\title{
Progress in Bright Ion Beams for Industry, Medicine and Fusion at LBNL
}

\author{
Joe W. Kwan \\ Lawrence Berkeley National Laboratory \\ I Cyclotron Road, Berkeley, CA 94546 USA
}

\begin{abstract}
Recent progresses at LBNL in developing ion beams for industry, radiation therapy and inertial fusion applications were discussed. The highlights include ion beam lithography, boron neutron capture therapy (BNCT), and heavy ion fusion (HIF) drivers using multiple linacs.
\end{abstract}

\section{INTRODUCTION}

Accelerator research at LBNL, in the Accelerator and Fusion Research Division, has a portfolio of diverse applications including areas outside high energy and nuclear physics such as semi-conductor industry, radiation therapy and fusion. This paper briefly reports the recent progress in the development of ion beam lithography, neutron capture radiation therapy, and heavy ion driven inertial fusion.

\section{SEMI-CONDUCTOR INDUSTRY}

It is well known that ion beams are useful for ion implantation in the semi-conductor industry. For this purpose, LBNL has developed ion sources such as the multi-cusp gas plasma sources and the Metal Vapor Vacuum Arc (MEVVA) sources. Another application that is under development is the ion projection lithography system.

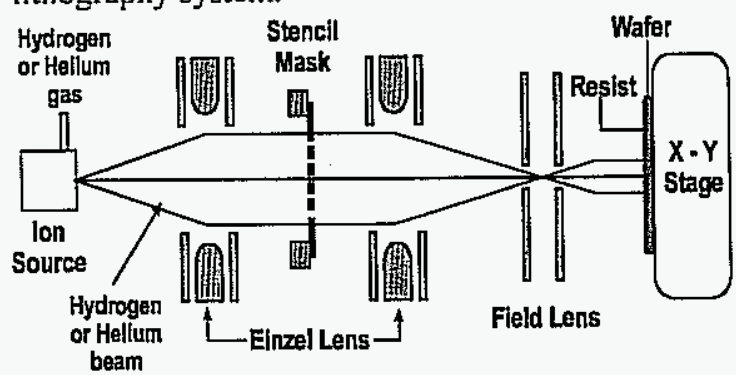

FIGURE 1. Ion projection lithography using a stencil mask.

In a conventional lithography system, a photon beam is projected through a stencil mask to produce images on a wafer that is covered by a layer of photoresist. As the industry advances, the dimension of components is now approaching the photon diffraction limit of $\sim 100 \mathrm{~nm}$. Ion beams can potentially extend the limit down to $\sim 5 \mathrm{~nm}$. Figure 1 shows a schematic of an ion projection lithography system by replacing the photon beam with an ion beam.
Nevertheless, constant ion bombardment on the stencil mask limits its lifetime, thus affecting both the replacement cost and downtime. A maskless microbeam reduction lithography system, as shown in Fig. 2, can improve the situation[1]. Here the exposure pattern is due to the aperture plate located at the ion source. The goal is to produce an array of microbeams with the ability to switch on each micro-beam individually.

Figure 3 shows the concept of constructing microbeam channels using layers of insulator. By applying

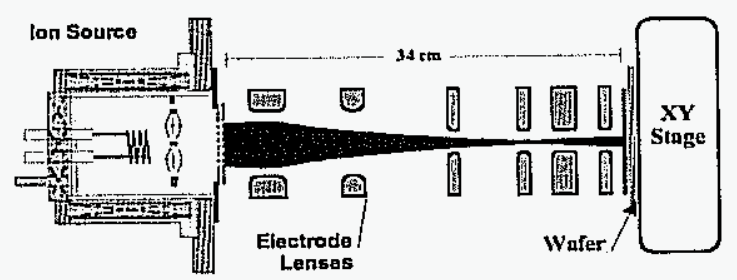

FIGURE 2. Maskless micro-beam reduction lithography.

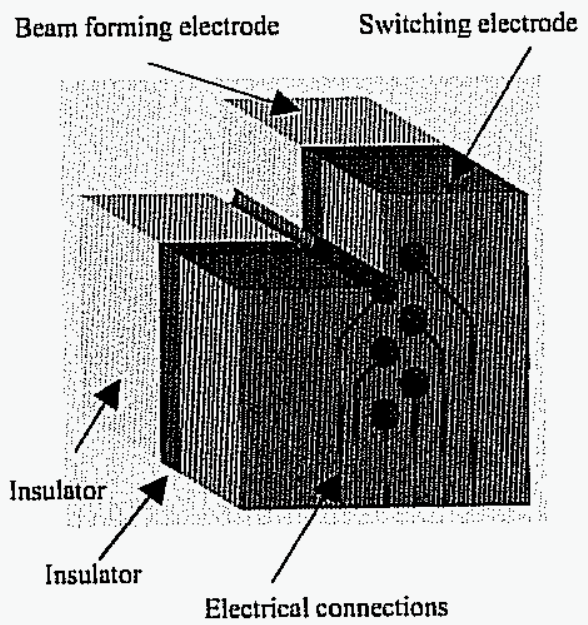

FIGURE 3. A micro-beam channel made of insulator layers. 
the appropriate voltages, a micro-beam can be gated either on or off. Separate layers of electrodes can be used to control rows of micro-beams in $x$ and $y$ directions for multiplexing.

\section{RADIATION THERAPY}

LBNL is a pioneer in radiation therapy since the early days of Bevatron. Nowadays proton and hadron therapies are common medical facilities worldwide. The trend is to develop facilities that are cost-effective, compact, and has good beam and dose controls. Figure 4 shows the size comparison of various types of machines. The new proposal is to build a $6-\mathrm{m}$ diameter super-conducting cyclotron for $250 \mathrm{MeV} / \mathrm{u}$ $(\mathrm{q} / \mathrm{m}=1 / 2)$ light ion therapy.

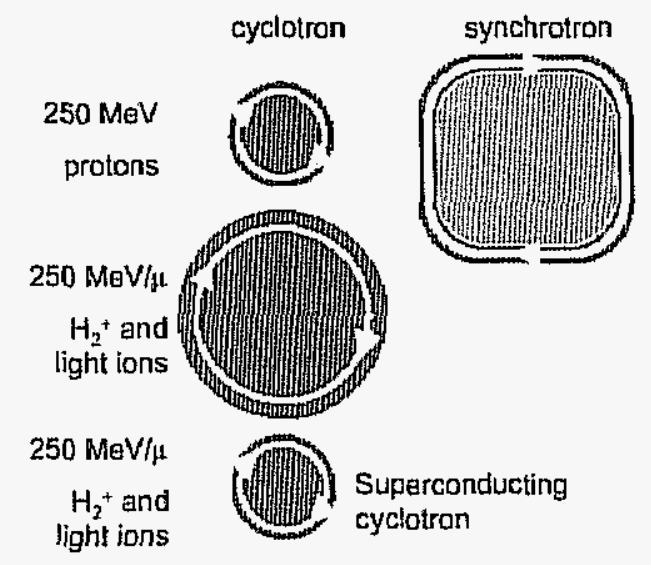

FIGURE 4. Comparing the sizes of various types of medical accelerators.

Another new challenge is to produce an accelerator-based neutron source for boron neutron capture therapy (BNCT). The concept of BNCT has been known for many years [2], but the practice lacks an effective boron-absorbing drug and neutron source of the correct energy spectrum. The basic principle of BNCT is to administer the patient with a tumorseeking chemical that contains boron. After a high concentration of boron is gathered at the tumor cells, the patient receives neutron radiation dose. Since the ${ }^{11} \mathrm{~B}$ formed by capturing a neutron is unstable and will decay into ${ }^{7} \mathrm{Li}+{ }^{4} \mathrm{He}$ with the emission of alpha radiation. The short-range alpha can destroy the tumor's DNA while leaving the surrounding normal cells unharmed by the epithermal neutrons. This type of treatment is most effective for glioblastoma brain tumors that are usually untreatable by other means.

Recently there is significant progress in developing tumor-seeking drugs with boron content e.g. BOPP. Hence a successful BNCT clinical trail is expected if we can provide the treatment with an appropriate neutron source. Figure 5 illustrates the difference in reactor-based and accelerator-based neutron energy spectrum. The most useful energy range for BNCT is between $1 \mathrm{keV}$ to $30 \mathrm{keV}$. These neutrons can be obtained by bombarding $2.5 \mathrm{MeV}$ protons on a $\mathrm{Li}$ target with a $\mathrm{Al} / \mathrm{AlF}_{3}$ moderator. The necessary dosage, to be delivered within a reasonable time $(\sim$ a few hours), requires a $20 \mathrm{~mA}$ dc proton beam.

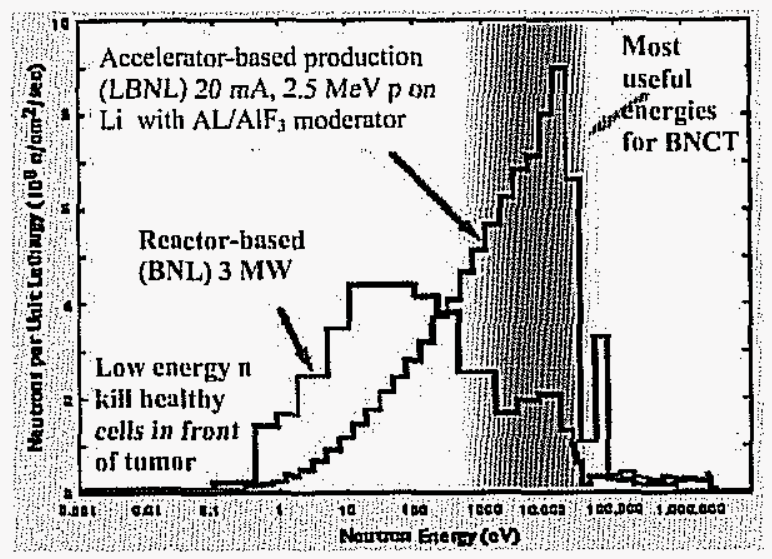

FIGURE 5. Accelerator-based neutron sources are superior to reactors for neutron therapy.

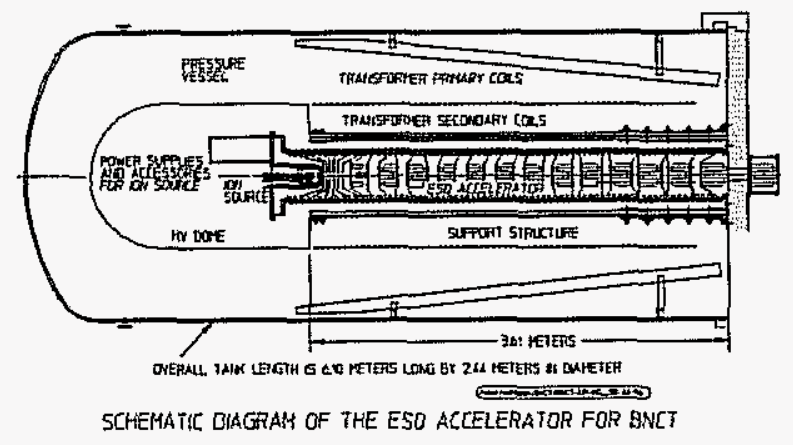

FIGURE 6, A $2.5 \mathrm{MV}, 50 \mathrm{~mA}$ proton de ESQ accelerator for BNCT; also similar design for contraband application.

As a spin-off technology from our fusion research, we have developed a conceptual design for a $2.5 \mathrm{MV}$, 50-100 mA proton dc accelerator [3]. Similar to a 2 MV injector used in the fusion program, the accelerator uses ESQ focusing to handle the beam's space charge force as well as stretching out the accelerator length to reduce longitudinal electric gradient (for preventing breakdowns). A schematic diagram of this accelerator is shown in Fig. 6.

The same accelerator design can be used for contraband applications. In that case a $1.76 \mathrm{MeV}, 10$ $\mathrm{mA}$ proton beam is directed onto a carbon target. The reaction ${ }^{13} \mathrm{C}(\mathrm{p}, \gamma){ }^{14} \mathrm{~N}$ releases the $9.17 \mathrm{MeV} \gamma$ radiation and subsequent resonant absorption by ${ }^{14} \mathrm{~N}$ will detect explosives and drugs [4]. 


\section{INERTIAL FUSION}

Ion beams are essential to progress in fusion research. There are two main approaches to fusion: magnetic confinement and inertial confinement. The most advanced magnetic confinement concept are the tokamak, a device that has a toroidal shape with magnetic field generated by field coils in combination with the self-field generated by the plasma current. Significant progresses have been made by injecting energetic deuterium beams to heat the plasma, drive current and control the density profile. These "neutral beam injectors" (developed by LBNL) typically have $100 \mathrm{keV}$ energy; the beam ions are converted into neutral particles before entering the tokamak. The new generation of tokamak is larger, thus requiring beams of up to $1 \mathrm{MeV}$ energy to penetrate the plasma.

While ion beams are auxiliary components in magnetic fusion devices, they are the main components in an inertial fusion machine using ion beam drivers. In comparison, ion beams can be more advantageous than laser beams because of the higher power efficiency $(-30 \%)$, higher duty rate $(\sim 10 \mathrm{~Hz})$ and more robust against radiation damages at the target chamber windows.

The goal of a heavy ion fusion driver is to deliver enough beam power to ignite a fusion target about 5 $\mathrm{mm}$ in diameter (see Fig.7 ). To do so, the required beam energy is $\sim 5 \mathrm{MJ}$ with a pulse length $\sim 10 \mathrm{~ns}$ in order to achieve a peak power of $\sim 10^{15} \mathrm{~W} / \mathrm{cm}^{2}$. Furthermore, the energy must be deposited within a short penetration range, e.g. 0.02 to $0.2 \mathrm{~g} / \mathrm{cm}^{2}$ of the target material. For heavy ions with atomic masses $\sim 200$, the allowable kinetic energy is $<10 \mathrm{GeV}$.

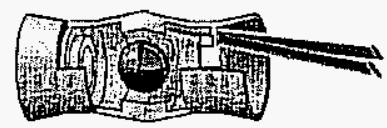

FIGURE 7. A typical IFE indirect-drive (hohlraum) target. Beam power is converted to $x$-ray radiation, compressing the target material to reach high density and ultimately high temperature for ignition.

Figure 8 is the block diagram of a typical heavy ion fusion driver using induction linacs [8]. Starting from a $2 \mathrm{MV}$ injector (that may contain up to 100 beams at $\sim 1$ A per beam), the ions will be accelerated to $\sim 100$ MV using electrostatic quadrupole (ESQ) focusing. At higher ion velocity, magnetic quadrupole focusing becomes more effective. Combining beams at this point may be preferred in order to optimize the overall cost and to rematch beams into the magnetic quadrupole lattices.

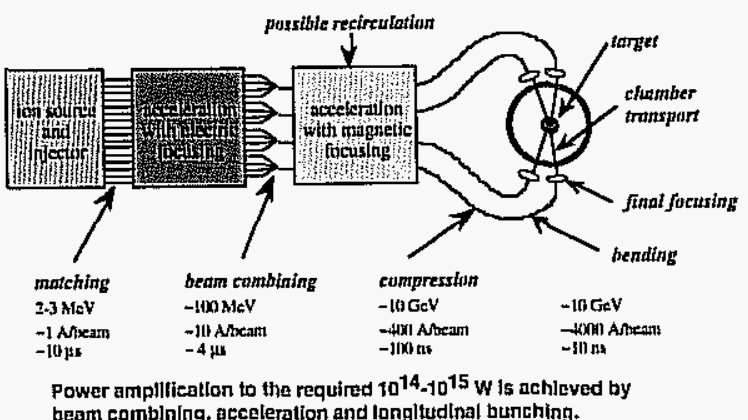

FIGURE 8. Block diagram of a typical Heavy Ion Beam Driver for IFE.

\section{Present HIF Experiments}

At present the HIF program is doing proof-ofprinciple experiments in order to learn how to build high current multiple beam injectors, how to transport space charge dominated beams through quadrupoles, and how to neutralized the space charge during final focusing into the target chamber. Figure 9 is a photograph of the High Current Experiment (HCX) at LBNL [6]. The goal is to transport $0.5 \mathrm{~A}$ of $\mathrm{K}^{+}$at 1.6 $\mathrm{MeV}$ energy through 30-40 ESQ modules and 4 or more magnetic quadrupoles in order to study the dynamic aperture, steering, scraping, emittance growth, and electron cloud effects.

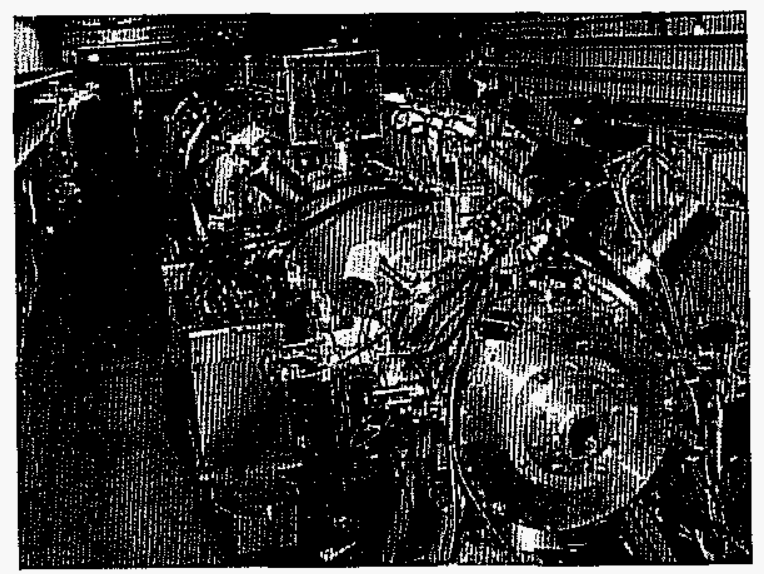

FIGURE 9. High Current Experiment (HCX) to study heavy ion beam transport at $0.25 \mu \mathrm{C} / \mathrm{m}$ line charge density.

To be cost effective, a fusion power plant driver must consist of multiple beams that share a common set of induction cores. Since the cost of the induction cores is a major cost factor, it is necessary to minimize the core size by building arrays of compact superconducting quadrupoles. Figure 10 is an example of how to pack multiple quadrupole channels.

The traditional HIF injector, as the one used in HCX shown in Fig. 9, is based on using a large diameter surface ionization source. This type of 
injector is considered too large (and too costly) when it comes to assembling $\sim 100$ beams for a driver. The large diameter beams also require a long matchingand-steering section that further complicates matter. Therefore we are developing a new type of compact injector that is based on using plasma ion sources coupled with mini-beamlet channels [7].

In the new design, more than a hundred of high intensity mini-beamlets are extracted and accelerated to $>1 \mathrm{MeV}$ inside individual channels before they are merged together to form a single beam of $0.5-1.0 \mathrm{~A}$ beam current ( $\mathrm{K}^{+}$equivalent). By controlling the aiming of beamlets, a merged beam can be quickly matched into the ESQ channel. Computer simulation (using a 3D PIC code) showed that the emittance of the merged beam was acceptable and the required brightness could be achieved if the mini-beamlets have current density $>100 \mathrm{~mA} / \mathrm{cm}^{2}$. Figure 11 gives a comparison of the size reduction between the traditional and the advanced designs.

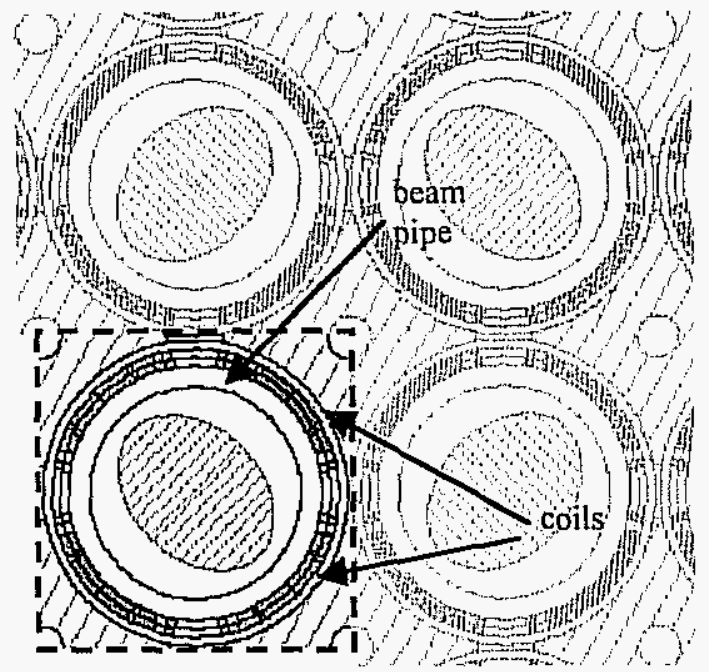

FIGURE 10. A HIF driver consists of an array of linacs using compact super-conducting quadrupoles.

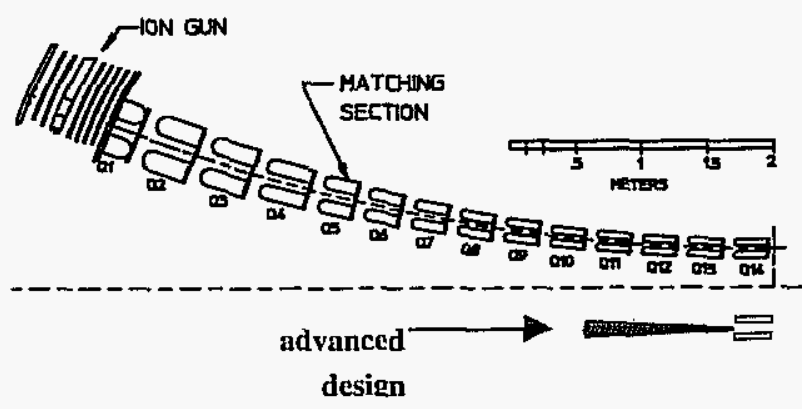

FIGURE 11. The HIF program is developing a new type of compact injector to replace the conventional type.

\section{Future HIF Projects and Plans}

The HIF program is proposing to build the next facility called the Integrated Beam Experiment (IBX), at about $\$ 50 \mathrm{M}$ total project cost. The experiment will be driver-scale in beam current but only short pulse $(\sim 1 \mu \mathrm{s})$ and limited in the number of parallel beams (1 upgradable to 4). This experiment will demonstrate integrated beam models for injection, acceleration, drift compression, final focus and chamber transport.

In our development path, on the way to eventually reaching a commercial IFE power plant, we expect another $\$ 300 \mathrm{M}$ facility containing prototype multiple beams ( $>4$ ) with full pulse length and beam energy at $\sim$ few $100 \mathrm{MeV}$. Successful demonstration of experiments on this facility will lead into the realization of a \$1B class DEMO at beam energies of a few $\mathrm{GeV}$.

\section{ACKNOWLEDGMENTS}

I would like to thank Dr. William Barletta for recomending me to give this invited talk, as well as his contribution to the viewgraphs. I am also thankful for the graphic materials provided by $\mathrm{Dr}$. Ka-Ngo Leung and Dr. Bill Chiu. This work is supported by the Office of Fusion Energy, US DOE under contract No. DE-AC03-76SF00098.

\section{REFERENCES}

1. Y. Lee, et al, Maskless Ion Beam Projection Lithography System, in Proc. the Intemational Conf. Micro- and Nano-Engineering, Septemer 22-24 1998, Leuven, Belgium.

2. Advances in Neutron Capture Therapy, Vol.l, Medicine and Physics, , Eds; B. Larsson, J. Crawford and R. Weinreich,(Elsevier Science B. V.), Amsterdam (1997).

3. J.W. Kwan et al., CP392, Application of Accelerators in Research and Industry, Eds: J. L. Duggan and I. L. Morgan, AIP Press, New York (1997) 1313.

4. S.T. Melnychuk, E. Kamykowski, J. Sredniawski, T. Debiak, R. Ruegg, B. Milton, in Proc. of the Particle Accelerator Conference, New York, 1999, p587.

5. R.O. Bangerter, in Proc. of Inter. Symp. on Heavy Ion Inertial Fusion, Frascati, Italy, 25-28 May, 1993, Nuovo Cimento, vol. 106A, N.11, pl445, (1993).

6. P. Seidl, et al, in Proc. of Inter. Symp. on Heavy Ion Inertial Fusion, Moscow, Russia, May 27-31, 2002, to be publjshed in Laser and Particle Beams.

7. J.W. Kwan, et al, Nucl. Instrum. and Methods (A), 464, p379, (2001)

Paper submitted to The 20th ICFA Advanced Beam Dynamics Workshop on High Intensity and High Brightness Hadron Beams, (ICFA-HB2002), April 8-12, 2002, Fermilab, USA 